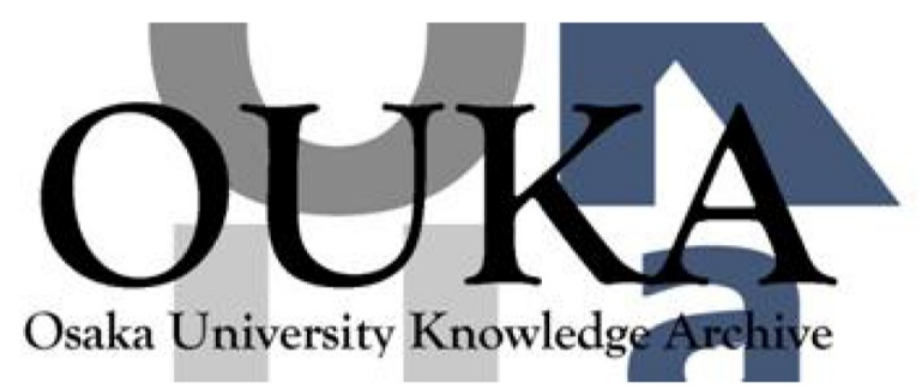

\begin{tabular}{|c|l|}
\hline Title & $\begin{array}{l}\text { Characterization of preplasma produced by an } \\
\text { ultrahigh intensity laser system }\end{array}$ \\
\hline Author(s) & Adumi, K.; Tanaka, K. A.; Matsuoka, T. et al. \\
\hline Citation & Physics of Plasmas. 11(8) p. 3721-p. 3725 \\
\hline Issue Date & $2004-08$ \\
\hline oaire:version VoR \\
\hline URL & https://hdl. handle. net/11094/3255 \\
\hline rights & \\
\hline Note & \\
\hline
\end{tabular}

Osaka University Knowledge Archive : OUKA

https://ir. Library. osaka-u. ac. jp/

Osaka University 


\title{
Characterization of preplasma produced by an ultrahigh intensity laser system
}

\author{
K. Adumi \\ Institute of Laser Engineering, Osaka University, 2-6 Yamada-oka, Suita, Osaka 565-0871, Japan \\ K. A. Tanaka \\ Institute of Laser Engineering, Osaka University, 2-6 Yamada-oka, Suita, Osaka 565-0871, Japan \\ and Faculty of Engineering, Osaka University, 2-1 Yamada-oka, Suita, Osaka 565-0871, Japan \\ T. Matsuoka, T. Kurahashi, T. Yabuuchi, Y. Kitagawa, R. Kodama, K. Sawai, K. Suzuki, \\ K. Okabe, T. Sera, T. Norimatsu, and Y. Izawa \\ Institute of Laser Engineering, Osaka University, 2-6 Yamada-oka, Suita, Osaka 565-0871, Japan
}

(Received 6 January 2004; accepted 20 April 2004; published online 21 June 2004)

\begin{abstract}
Ultra intense laser interaction with solids is crucially modified by the presence of the plasma layer created by the prepulse associated with the peak intensity pulse. Here interferometric measurements of the preplasma created by a $40 \mathrm{TW}, 600 \mathrm{fs}$ Nd-glass laser are reported. Density profile and its target dependence are measured and the predicted scale length dependence on target atomic number is confirmed. (C) 2004 American Institute of Physics. [DOI: 10.1063/1.1760774]
\end{abstract}

\section{INTRODUCTION}

The interaction of very intense, ultrashort laser pulses with matter is a very exciting contemporary area of research that not only addresses many basic physics issues but also offers potential applications in fast ignition of inertial confinement fusion, ${ }^{1-4}$ novel x-ray, gamma ray, ${ }^{5-9}$ and charged particle sources, ${ }^{10-13}$ and so on. The state of the art peak intensities today are pegged at $10^{21} \mathrm{~W} / \mathrm{cm}^{2},{ }^{14}$ which are five orders of magnitude larger than the atomic unit of intensity. The direct application of such light intensities to ordinary matter is, however, complicated by the fact that the pulses are not really ultrashort but have significant prepulses. ${ }^{15}$ The higher the main peak intensity, the higher is the prepulse level. These prepulses arise either in the form of independent laser pulses or as pedestals of the main laser pulse. They typically occur a few picoseconds/nanoseconds before the main, subpicosecond pulse and effectively increase the pulse duration at high light intensities. For example, with a peak of $10^{21} \mathrm{~W} / \mathrm{cm}^{2}$, and a reasonably good main pulse to prepulse contrast of $10^{6}$, we still have $10^{15} \mathrm{~W} / \mathrm{cm}^{2}$ in the picosecond prepulse of a femtosecond main pulse. This is too large an intensity for normal matter to survive unionized and leads to the formation of a preplasma. ${ }^{16}$ It is now well established that the preplasma has a crucial role to play in the absorption of the main laser pulse and can also aid the formation of plasma instabilities, and so on. For instance, the process of resonance absorption (RA) is not very efficient for very short, clean (prepulse free) laser pulses, as it needs an optimum plasma scale length. Many experiments at lower intensities create an intentional preplasma to aid RA and increase the resultant hot electron generation and $\mathrm{x}$-ray emission. ${ }^{17,18}$ On the other hand, absorption mechanisms like vacuum heating are rendered ineffective at long plasma scale lengths and, therefore, preplasma could reduce such coupling. In very high intensity experiments, however, the strong preplasma created could affect the physics very significantly by chang- ing the coupling, triggering plasma instabilities and altering the intended features of the experiment and its interpretation. Recent experiments have claimed that preplasma also depends on the target material rather dramatically - plastic targets show preplasma at intensities as low as $10^{9} \mathrm{~W} / \mathrm{cm}^{2} .{ }^{19} \mathrm{It}$ is therefore very essential to characterize the preplasma carefully and establish its dependence on target and laser conditions. The correct interpretation of these experiments is possible only with the knowledge of the preplasma. It is, however, found that many experiments reported in the literature merely mention or allude to the preplasma, without specifying its characteristics. In fact, there is a relatively small number of detailed reports on preplasma measurements.

The Gekko Module II (GMII) Nd-glass laser at the Institute of Laser Engineering, Osaka University, a typical high intensity laser, is used for many experiments on laserproduced plasmas at relativistic intensities (above $10^{18} \mathrm{~W} / \mathrm{cm}^{2}$ ). Its high peak power is obtained by the chirped pulse amplification (CPA) technique, which is known to produce pulses with prepulses due to amplified spontaneous emission in the amplifiers and incomplete compression of the pulses. It is very essential to establish the role of preplasma in these experiments so that a correct interpretation of the experimental results is made. In this paper, we characterize the preplasma produced by this laser using an interferometric technique. We establish the scale length of the electron density profile and its dependence on the main peak intensity and target atomic number. These results should be useful for similar studies of laser-produced plasmas at these high intensities.

\section{EXPERIMENT}

\section{A. The laser system}

The GMII laser produces $600 \mathrm{fs}, 1.053 \mu \mathrm{m}$ laser pulses at a low repetition rate. The maximum pulse energy is $20 \mathrm{~J}$ 
giving a peak power of nearly $40 \mathrm{TW}$ and an intensity of $10^{18} \mathrm{~W} / \mathrm{cm}^{2}$. A portion of the laser pulse is extracted to measure the prepulse level. It is monitored by a PositiveIntrinsic-Negative (PIN) diode coupled to an oscilloscope for every shot. The cut-off frequency of the PIN diode (Hamamatsu, G3476-01) is $2 \mathrm{GHz}$ and its dynamic range is about $10^{2}$. Appropriate, calibrated neutral density filters were used to attenuate high energy levels to below the saturation limit of the PIN diode. Two prepulses, which precede the high intensity main pulse by (1) 700 ps and (2) $6.5 \mathrm{~ns}$, respectively, can be detected. The uncertainty in the sampling rate is $200 \mathrm{ps}$. The prepulses originate in the regenerative amplifier of the laser system and can be assumed to have the same chirp levels. They are, therefore expected to have the same duration as the main pulse after compression. The energies of the prepulses are known from the output voltage of the PIN diode using a calibration value. Prepulse (1) has an energy that is $3 \times 10^{-3}$ that of the main pulse, while prepulse (2) has $10^{-4}$ times the main pulse energy. The effect of prepulse (2) can be neglected because it is only $3 \%$ as energetic as prepulse (1). Prior to prepulse (1), 700 ps before the main pulse, the intensity of amplified spontaneous emission (ASE) is less than $10^{-8}$ times smaller than main pulse, which is the detection limit. ${ }^{22}$ The intensity of prepulse (1) focused on the target surface is about $10^{15} \mathrm{~W} / \mathrm{cm}^{2}$ and it generates the preplasma by the interaction with the target.

\section{B. Interferometry}

Interferometry is a standard technique for measuring electron density profiles. $^{20,21}$ It relies on the phase shift experienced by a probe beam as it traverses the plasma. The phase shift is caused by the variation of the refractive index in the plasma as a function of density. It is well known that the refractive index of the plasma $\mu$ is a function of the electron density and is given by

$$
\mu=\sqrt{1-\frac{n_{e}}{n_{c}}},
$$

where $n_{e}$ is the electron density and $n_{c}$ is critical density. If $n_{e} \ll n_{c}$, the above equation can be approximated as

$$
\mu=1-\frac{n_{e}}{2 n_{c}} .
$$

We assume that there is negligible absorption and refraction of the probe light in the plasma, and that the plasma has cylindrical symmetry with the axis of the direction perpendicular to target surface. Figure 1 shows the coordinate frame in the plasma. The optical path length of the light propagating in the plasma $l(x)$ becomes

$$
l(x)=2 \int_{x}^{r_{0}} \frac{n(r) r}{\sqrt{r^{2}-x^{2}}} \mathrm{~d} r .
$$

Here $n(r)$ is the electron density. Optical path length of the light in vacuum, $l_{0}(x)$, is

$$
l_{0}(x)=2 \sqrt{r_{0}^{2}-x^{2}} .
$$

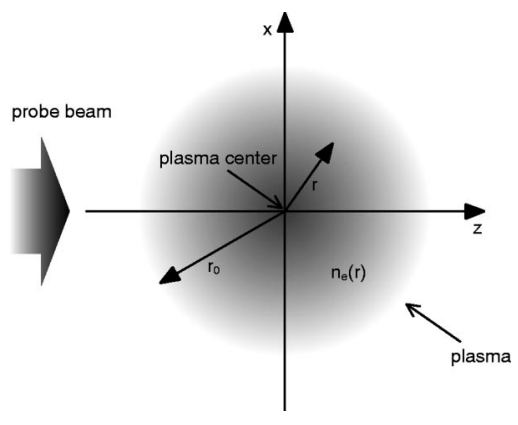

FIG. 1. Cylindrically symmetric plasma.

Therefore, the difference of length between the paths in vacuum and in plasma is

$$
l(x)-l_{0}(x)=-\frac{1}{n_{c}} \int_{x}^{r_{0}} \frac{n_{e}(r) r}{\sqrt{r^{2}-x^{2}}} \mathrm{~d} r .
$$

The phase shift becomes

$$
\phi(x)=-\frac{2 \pi}{\lambda n_{c}} \int_{x}^{r_{0}} \frac{n_{e}(r) r}{\sqrt{r^{2}-x^{2}}} \mathrm{~d} r .
$$

The above equation is a form of the Abel transform. ${ }^{23-25}$ Thus, electron density is obtained by applying Abel inversion, like

$$
n_{e}(r)=\frac{\lambda n_{c}}{\pi^{2}} \int_{r}^{r_{0}} \frac{\mathrm{d} \phi(x)}{\mathrm{d} x} \frac{1}{\sqrt{x^{2}-r^{2}}} \mathrm{~d} x .
$$

We can obtain the phase shift of the probe beam from the interferogram by using fast Fourier transform techniques. ${ }^{26} \mathrm{~A}$ fringe pattern of interferogram can be described as

$$
g(x, y)=a(x, y)+b(x, y) \cos \left[2 \pi f_{0} x+\phi(x, y)\right],
$$

where $f(x, y)$ is phase shift, $a(x, y)$ and $b(x, y)$ are unwanted irradiance variations, and $f_{0}$ is the spatial-carrier frequency. This equation can be rewritten as

$$
\begin{aligned}
g(x, y)= & a(x, y)+c(x, y) \exp \left(i 2 \pi f_{0} x\right) \\
& +c^{*}(x, y) \exp \left(-2 \pi i f_{0} x\right)
\end{aligned}
$$

with

$$
c(x, y)=\frac{1}{2} b(x, y) \exp [i \phi(x, y)]
$$

where $*$ denotes a complex conjugate. Equation (10) is Fourier transformed with respect to $x$ and becomes

$$
G(f, y)=A(f, y)+C\left(f-f_{0}, y\right)+C^{*}\left(f+f_{0}, y\right),
$$

where the capital letters denote the Fourier spectra and $f$ is the spatial frequency in the $x$ direction. Because the spatial variations of $a(x, y), b(x, y)$, and $f(x, y)$ are slow enough compared with $f_{0}, C\left(f-f_{0}, y\right)$ can be extracted. $C(f$ $\left.-f_{0}, y\right)$ is moved by $f_{0}$ in parallel to the frequency axis to obtain $C(f, y)$. Then $C(f, y)$ is inverse Fourier transformed to obtain $c(x, y)$. So we can calculate the phase shift, 


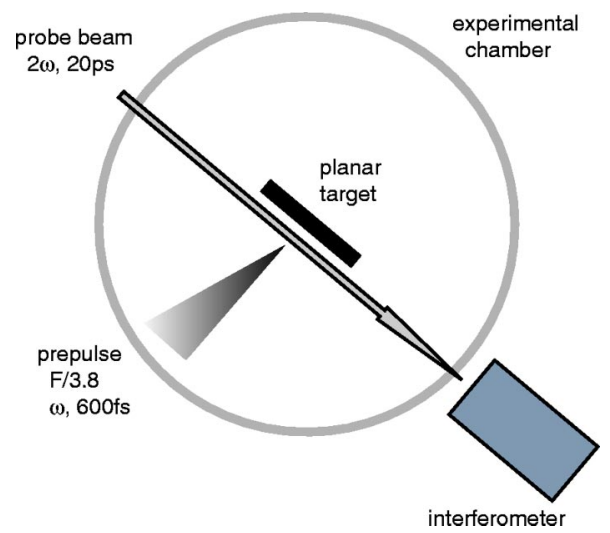

FIG. 2. Experimental setup (top view).

$$
\phi(x, y)=\arctan \left[\frac{\mathfrak{R}[c(x, y)]}{\mathfrak{I}[c(x, y)]}\right] .
$$

Therefore we can obtain the electron density substituting $\phi(x, y)$ for Eq. (7).

\section{Interferometry setup}

The experimental setup is shown in Fig. 2. Planar targets of $20 \mu \mathrm{m}$ thickness were used. This thickness is much larger than the skin depth for laser light. The targets were made of three materials, $\mathrm{Au}, \mathrm{Al}$ and deuterated carbon $(\mathrm{CD})$. The prepulse is focused on the target surface by an F/3.8 off-axis parabola mirror, and the preplasma is generated. The wavelength of the prepulse is $1.053 \mu \mathrm{m}(1 \omega)$, and the pulse duration is $600 \mathrm{fs}$, and the laser intensity is of the order of $10^{15} \mathrm{~W} / \mathrm{cm}^{2}$. We have used a shearing interferometer ${ }^{21}$ to measure electron density profile of the plasma. A separate probe beam passes the preplasma parallel to the target surface $50 \mathrm{ps}$ before the main laser pulse hits the target surface. The wavelength of the probe light is $0.527 \mu \mathrm{m}(2 \omega)$, and the pulse duration, $20 \mathrm{ps}$. The interferogram is observed by a charge-coupled device (CCD) camera (see Fig. 3). This figure depicts the data obtained for the Al target and the two-

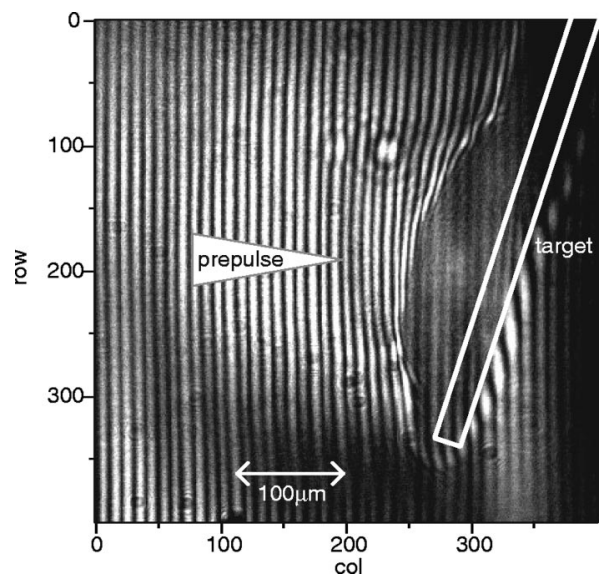

FIG. 3. An interferogram in the case of $\mathrm{Al}$ target. A prepulse comes from the left-hand side.

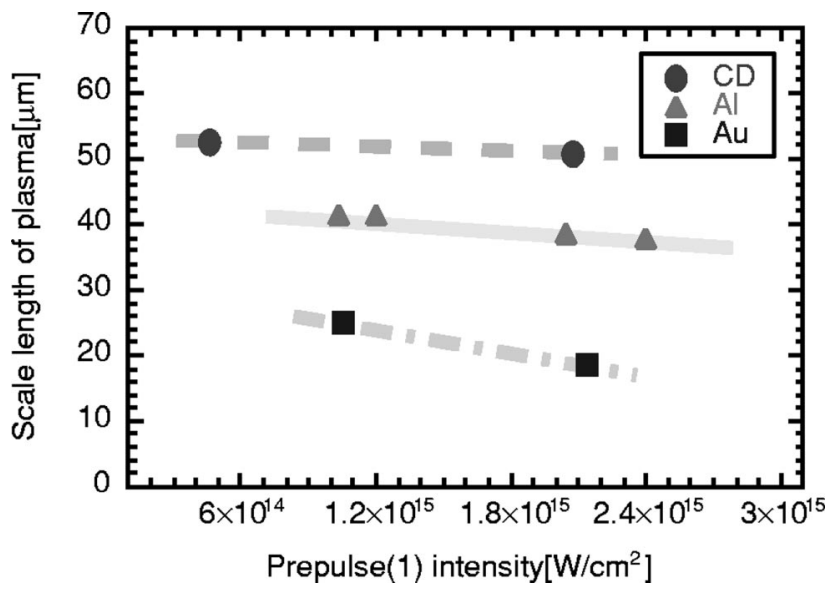

FIG. 4. Variation of plasma scale length with prepulse (1) intensity. The intensity of ASE is weaker than main pulse by a factor of $10^{-8}$.

dimensional electron density distribution is obtained by analyzing this image. The spatial resolution of the interferogram is $5.9 \mu \mathrm{m}$.

\section{RESULTS AND DISCUSSIONS}

The two-dimensional electron density profile in the lowdensity region of about $1.0 \times 10^{19}-5.0 \times 10^{19} / \mathrm{cm}^{3}$ can be obtained from the interferogram. We extracted a onedimensional electron density profile in the perpendicular direction to the target in order to examine target material dependence of the plasma expansion and deduced the scale length of the plasma from that. Here, to obtain the scale length, the electron density profile is fitted by an exponential function, and the following definition of scale length $L$ is used:

$$
L=\left[\frac{1}{n_{e}}\left(\frac{\mathrm{d} n_{e}}{\mathrm{~d} x}\right)\right]^{-1} .
$$

The relationship between the scale length obtained from the experiment and intensity of the prepulse is shown in Fig. 4. The atomic number of $\mathrm{CD}$ was defined as the mean value of atomic number of carbon and deuterium. It is evident from this figure that there is no change in the scale length, even if the prepulse intensity changes by a factor of 2 . The scale lengths for $\mathrm{CD}, \mathrm{Al}$, and $\mathrm{Au}$ are $50 \mu \mathrm{m}, 40 \mu \mathrm{m}$, and $20 \mu \mathrm{m}$ respectively. It can be seen that the scale length shortens as the target atomic number increases. In Fig. 5 the typical electron density profile of plasmas in the case of $\mathrm{CD}$ and $\mathrm{Au}$ targets is shown. Evidently, CD plasma has a larger scale length than the Au plasma.

Let us now consider isothermal expansion of an ideal plasma. Assuming self-similarity, the speed at which the plasma expands is given by

$$
c_{s}=\sqrt{\frac{Z T_{e}}{M}} \simeq \sqrt{\frac{Z T_{e}}{A m_{p}}},
$$

where $Z$ is the ion charge state, $T_{e}$ is an electron temperature, $M$ is a mass of the ion, $A$ is mass number, and $m_{p}$ is a mass of a proton. $T_{e}$ tends to be low in the case of targets with high atomic number due to the large ionization energy. As far 


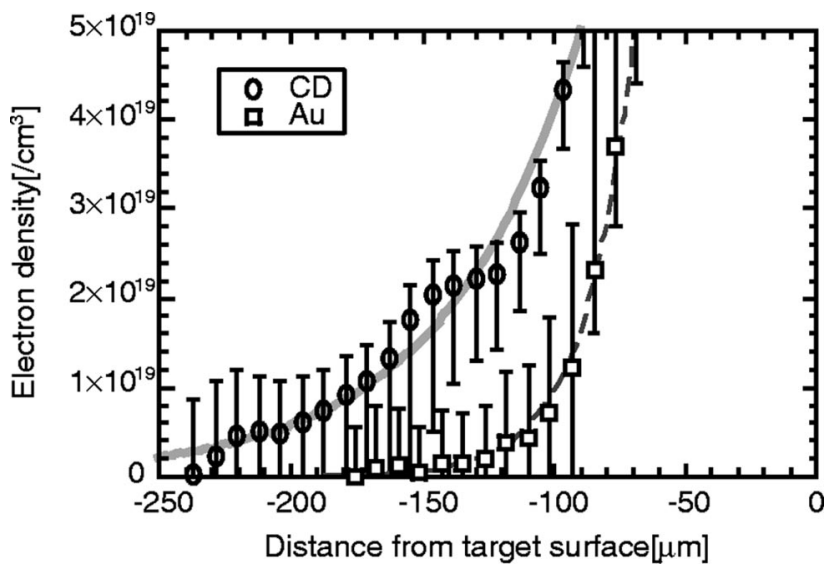

FIG. 5. Comparison of electron density profile of the plasmas between CD target (circle) and Au target (square). The solid line and dashed line are approximated curves.

as $Z / A$ is concerned, if atoms of a target material are ionized fully, its value does not change significantly and is $\sim 0.5$. However, for our prepulse intensity, only low atomic number atoms may be fully ionized. Therefore, under these conditions, $Z / A$ is rapidly reduced as the atomic number of target material increases. In this model the relationship between the plasma expansion speed and the scale length of the plasma is $L=c_{s} t$, where $t$ is the time elapsed from the instant the laser hits the target. Thus, we can understand that plasma expansion speed slows down, as the target atomic number increases and, hence, the scale length decreases for higher atomic number.

We simulated our experiment using the one dimensional hydrodynamic code "ILESTA-1D." ${ }^{27} T_{e}$ and $Z$ of the preplasma can be obtained from this simulation results. A plasma expansion speed $c_{s}$ is calculated by using $T_{e}$ and $Z$ right after irradiation. The relationship between the value $c_{s}$ calculated from this simulation results and the experimental scale length of plasma $L$ is shown in Fig. 6. The scale length $L$ is obviously proportional to the plasma expansion speed $c_{s}$. This tendency corresponds to the above isothermal

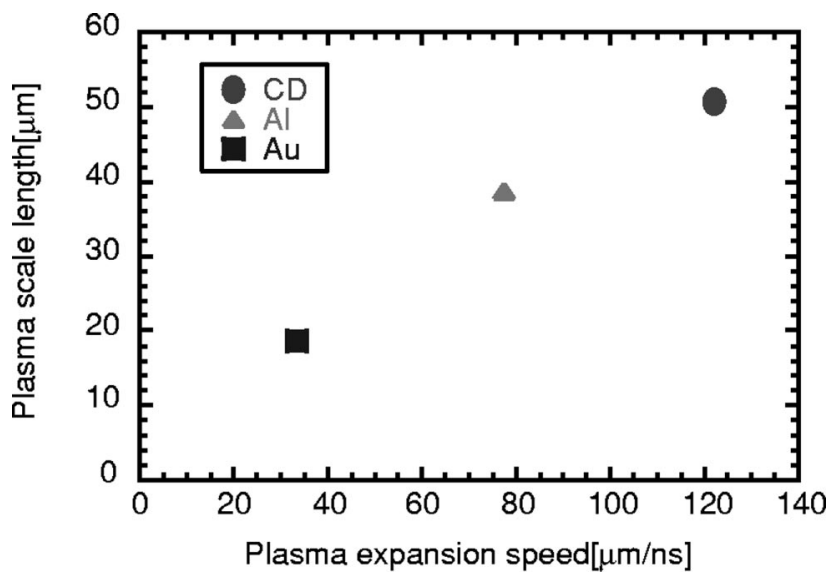

FIG. 6. The relationship between the value $c_{s}$ obtained from this simulation and the experimental scale length of plasma. The plasma scale length $L$ is proportional to the plasma expansion speed $c_{s}$ calculated by using the simulation results. plasma expansion model, in which plasma expands at constant speed $c_{s}$. Hence we can explain the plasma expansion with this simple model.

\section{CONCLUSION}

In summary, the scale length of the plasma created by the prepulse is measured in three different materials with quite different atomic numbers. We confirmed that the plasma with the scale length of $20-50 \mu \mathrm{m}$ is generated at intensities of $10^{15} \mathrm{~W} / \mathrm{cm}^{2}$. We demonstrate that the scale length of the plasma shortens with the increase of the atomic number as expected.

\section{ACKNOWLEDGMENTS}

The authors wish to thank Professor G. Ravindra Kumar for useful comments and discussions. They would also like to acknowledge Target group at the Institute of Laser Engineering for target fabrication.

${ }^{1}$ M. Tabak, J. Hammer, M. E. Glinsky, W. L. Kruer, and S. C. Wilks, Phys. Plasmas 1, 1626 (1994).

${ }^{2}$ R. Kodama, P. A. Norreys, K. Mima, A. E. Dangor, R. G. Evans, H. Fujita, Y. Kitagawa, K. Krushelnick, T. Miyakoshi, N. Miyanaga, T. Norimatsu, S. J. Rose, T. Shozaki, K. Shigemori, A. Sunahara, M. Tampo, K. A. Tanaka, Y. Toyama, T. Yamanaka, and M. Zepf, Nature (London) 412, 798 (2001).

${ }^{3}$ R. Kodama, H. Shigemori, Y. Toyama, S. Fujioka, H. Fujioka, H. Azechi, H. Fujita, H. Habara, T. Hall, Y. Izawa, T. Jitsuno, Y. Kitagawa, K. M. Krushelnick, K. L. Lancaster, K. Mima, K. Nagai, M. Nakai, H. Nishimura, T. Norimatsu, P. A. Norreys, S. Sakabe, K. A. Tanaka, A. Youssef, M. Zepf, and T. Yamanaka, Nature (London) 418, 933 (2002).

${ }^{4}$ K. A. Tanaka, R. Kodama, K. Mima, Y. Kitagawa, H. Fujita, N. Miyanaga, K. Nagai, T. Norimatsu, T. Sato, Y. Sentoku, K. Shigemori, A. Sunahara, T. Shozaki, M. Tanpo, S. Tohyama, T. Yabuuchi, J. Zheng, T. Yamanaka, P. A. Norreys, R. Evanse, M. Zepf, K. Krushelnic, A. Dangor, R. Stephens, S. Hatchett, M. Tabak, and R. Turner, Phys. Plasmas 10, 1925 (2003).

${ }^{5}$ M. M. Murnane, H. C. Kapteyn, and R. W. Falcone, Phys. Fluids B 3, 2409 (1991).

${ }^{6}$ J. D. Kmetic, C. L. Gordon III, J. J. Macklin, B. E. Lemoff, G. S. Brown, and S. E. Harris, Phys. Rev. Lett. 68, 1527 (1992).

${ }^{7}$ J. Workman, A. Maksimchuk, X. Liu, U. Ellenberger, J. S. Coe, C.-Y. Chien, and D. Umstadter, Phys. Rev. Lett. 75, 2324 (1995).

${ }^{8}$ K. A. Tanaka, Rev. Laser Eng. 29, 238 (2001).

${ }^{9}$ P. P. Rajeev, P. Taneja, P. Ayyub, A. S. Sandhu, and G. Ravindra Kumar, Phys. Rev. Lett. 90, 115002 (2003).

${ }^{10}$ T. Tajima and J. M. Dawson, Phys. Rev. Lett. 43, 267 (1979).

${ }^{11}$ K. B. Wharton, S. P. Hatchett, S. C. Wilks, M. H. Key, J. D. Moody, V. Yanovsky, A. A. Offenberger, B. A. Hammel, M. D. Perry, and C. Joshi, Phys. Rev. Lett. 81, 822 (1998).

${ }^{12}$ S. C. Wilks, A. B. Langdon, T. E. Cowan, M. Roth, M. Singh, S. Hatchett, M. H. Key, D. Pennington, A. MacKinnon, and R. A. Snavely, Phys. Plasmas 8, 542 (2001).

${ }^{13}$ K. W. D. Ledingham, P. McKenna, and R. P. Singhal, Science 300, 1107 (2003).

${ }^{14}$ M. D. Perry, D. Pennington, B. C. Stuart, G. Tietbohl, J. A. Britten, C. Brown, S. Herman, B. Golick, M. Kartz, J. Miller, H. T. Powell, M. Vergino, and V. Yanovsky, Opt. Lett. 24, 160 (1999).

${ }^{15}$ J.-C. Diels and W. Rudolph, Ultrashort Laser Pulse Phenomena (Academic, San Diego, 1996).

${ }^{16}$ E. G. Gamaly, A. V. Rode, B. Luther-Davies, and V. T. Tikhonchuk, Phys. Plasmas 9, 949 (2002).

${ }^{17}$ H. Nakano, T. Nishikawa, H. Ahn, and N. Uesugi, Appl. Phys. Lett. 69, $2992(1996)$.

${ }^{18}$ S. Bastiani, A. Rousse, J. P. Geindre, P. Audebert, C. Quoix, G. Hamoniaux, A. Antonetti, and J.-C. Gauthier, Phys. Rev. E 56, 7179 (1997).

${ }^{19}$ K. B. Wharton, C. D. Boley, A. M. Komashko, A. M. Rubenchik, J. Zweiback, J. Crane, G. Hays, T. E. Cowan, and T. Ditmire, Phys. Rev. E 64, $025401(2001)$ 
${ }^{20}$ J. A. Cobble, R. P. Johnson, N. A. Kurnit, D. S. Montgomery, and J. C. Fernandéz, Rev. Sci. Instrum. 73, 3813 (2002).

${ }^{21}$ I. H. Hutchinson, Principles of Plasma Diagnostics (Cambridge University Press, Cambridge, 2002).

${ }^{22}$ M. Mori, Ph.D. thesis, Faculty of Engineering, Osaka University, 2000.

${ }^{23}$ M. Deutsch and I. Beniaminy, Appl. Phys. Lett. 41, 27 (1982).

${ }^{24}$ P. A. Vicharelli and W. P. Lapatovich, Appl. Phys. Lett. 50, 557 (1987).
${ }^{25}$ S. Gueron and M. Deutsch, J. Appl. Phys. 75, 4313 (1994).

${ }^{26}$ M. Takeda, H. Ina, and S. Kobayashi, J. Opt. Soc. Am. 72, 156 (1982).

${ }^{27}$ H. Takabe, M. Yamanaka, K. Mima, C. Yamanaka, H. Azechi, N. Miyanaga, M. Nakatsuka, T. Jitsuno, T. Norimatsu, M. Takagi, H. Nishimura, M. Nakai, T. Yabe, T. Sasaki, K. Yoshida, K. Nishihara, Y. Kato, Y. Izawa, T. Yamanaka, and S. Nakai, Phys. Fluids 31, 2884 (1988). 